\title{
Is Airwayscope more effective than Macintosh laryngoscope?
}

\author{
Xin Long Cui $\cdot$ Fu Shan Xue $\cdot$ Rui Ping Li
}

Received: 5 January 2014/ Accepted: 14 January 2014/Published online: 25 February 2014

(C) Japanese Society of Anesthesiologists 2014

Keywords Airway management · Tracheal intubation · Airway devices

To the Editor:

In a manikin study comparing the Pentax-AWS Airwayscope (AWS) and the Macintosh laryngoscope (MLS) during chest compressions, Kohama et al. [1] showed that the AWS was superior to the MLS. Also, they reported the same results in previous manikin studies simulating chest compressions. However, their results are not supported by a randomized controlled trial in prehospital patients primarily with cardiac arrest, in which the AWS was inferior to the MLS in intubation time, success rate, and difficulty of intubation [2].

In this clinical study, an important cause of failed intubation with AWS on the first attempt is oral contamination, which occurs in 45 of 109 patients. When the AWS lens is obscured by contamination, it needs to be removed and cleaned. Obviously, this requires extra time and affects intubation time and success rate. During intubation with MLS, however, use of a suction device to clean oral contamination is easy, and the MLS does not need to be removed to clean the contamination. Actually, vomitus or secretions cannot be modeled by simple or even sophisticated manikins. Rai and Popat [3] have pointed out that manikin studies often reveal results that are impossible to interpret or are even contradictory to subsequent human studies. Thus, we wish to echo Behringer and Kristensen [4] that manikin studies are of negligible value as sole predictor of any given airway device's value in the clinical setting.

Conflict of interest The authors have no competing interests to declare.

\section{References}

1. Kohama H, Komasawa N, Ueki R, Yamamoto N, Tashiro C, Kaminoh Y, Nishi S. Utility of the Pentax-AWS Airwayscope and Macintosh laryngoscope for airway management during chest compressions in 27 left-lateral tilt: a manikin simulation study of maternal cardiopulmonary resuscitation. J Anesth. 2013;27:671-5.

2. Arima T, Nagata O, Miura T, Ikeda K, Mizushima T, Takahashi A, Sakaida K. Comparative analysis of Airwayscope and Macintosh laryngoscope for intubation primarily for cardiac arrest in prehospital setting. Am J Emerg Med. 2014;32:40-3.

3. Rai MR, Popat MT. Evaluation of airway equipment: man or manikin? Anaesthesia. 2011;66:1-3.

4. Behringer EC, Kristensen MS. Evidence for benefit vs. novelty in new intubation equipment. Anaesthesia. 2011;66(suppl 2):57-64.

An answer to this letter to the editor is available at doi:10.1007/s00540-014-1800-3.

X. L. Cui · F. S. Xue $(\varangle)$ · R. P. Li

Department of Anesthesiology, Plastic Surgery Hospital,

Chinese Academy of Medical Sciences and Peking Union

Medical College, 33 Ba-Da-Chu Road, Shi-Jing-Shan District,

Beijing 100144, People's Republic of China

e-mail: xuefushan@aliyun.com; fushan.xue@gmail.com 